\title{
1.5-multiplicity molecular light scattering in fluids?
}

\author{
M.Ya.Sushko* \\ Odesa Mechnikov University, \\ 2 Dvoryanska St., Odesa 65026, Ukraine
}

Received June 25, 2005

The third-order thermodynamic moment of the density fluctuations is shown to contribute notably to the molecular light scattering in a single-component fluid. The relevant 1.5-scattering is expected to affect the depolarization ratio of the scattered light as the scattering is studied near the critical isochore and for the temperature interval where nonlocal correlation between fluctuations is relatively weak. The experimental data in support of this conclusion are quoted.

Key words: density fluctuations, third moment, light scattering

PACS: $05.40,05.70 . F h, 64.70 . F x, 78.35 .+C$

\section{Introduction}

The analysis of the molecular light scattering data for fluids near the critical point is usually carried out in terms of the single and double scattering mechanisms. Systematic studies of the latter were initiated by different authors [1-5] three decades ago and soon resulted in its major features being well-established (see, for instance, a comprehensive review [6]). In addition, the triple scattering effects were also reported to be observed [7].

Since the appearance of [6], the basic approach to the problem has virtually remained intact. The attention is focused on the true double scattering caused by successive single scatterings off atoms or density fluctuations that are separated by macroscopic distances $r \gg \lambda \gtrsim r_{\mathrm{c}}, \lambda$ and $r_{\mathrm{c}}$ being the wavelength in the medium and the correlation length respectively. This wave-zone condition enables one to use the asymptotes $[8,9]$ for the four-point correlation functions and concentrate on evaluating the "ladder term" yielding the dominant contribution. As for the so-called 1.5-scattering, the known estimations with the help of similar approximations (such as [1,2], where the Kirkwood superposition approximation and asymptotes [8] were employed) have led to the commonly accepted conclusion that it is impossible to detect.

In this paper, we report the results and further development of our attacks $[10,11]$ on the 1.5scattering mechanism, which were made without referring to any uncouplings. We believe that the use of different uncouplings for the three- and four-point correlation functions is equivalent to the adoption of a Gaussian-like model, where 1.5-scattering is negligible and no new information of higher-order correlation functions is available. It follows that only those three-point configurations where all the scattering centres are the distances $r \lesssim r_{\mathrm{c}}<\lambda$ apart from one another (so that the three-point correlation functions cannot be uncoupled) may contribute significantly to the scattering. It should be noted that this condition corresponds to the temperature interval $\tau \equiv$ $\left|T-T_{\mathrm{c}}\right| / T_{\mathrm{c}} \gtrsim 10^{-5}$, typical of actual experiment.

Our approach can be summarized as follows. We use a macroscopic description and analyze the short-range singularities of the electromagnetic field propagator to extract the leading compactgroup contributions to the total polarized scattering intensity $I$ from each member of the iteration series for the scattered field. In the immediate vicinity of the critical point, the expression for $I$ is in accordance with the hypothesis of the algebra of fluctuating quantities [12]. At a certain distance from the critical point, where nonlocal correlation between fluctuations is weak, the individual

*E-mail: mrs@onu.edu.ua 
members in the series for $I$ are expressed through the second and higher-order thermodynamic moments of density fluctuations. In particular, the 1.5-scattering contribution is related to the third (non-Gaussian) moment.

The analysis with the use of the grand canonical ensemble and the van der Waals equation reveals that the contribution to the permittivity fluctuations from this moment may exceed that from the Gaussian part of the fourth moment. The temperature and density ranges are determined where the 1.5-scattering is of the largest relative value and can exceed the true double scattering. The fact that the 1.5-scattering intensity has a negative sign for positive values of the parameter $\omega \equiv \rho / \rho_{\mathrm{c}}-1$, describing the deviation of the density $\rho$ from the critical value $\rho_{\mathrm{c}}$, favours its separation from the total scattering intensity. In particular, the 1.5-scattering is expected to affect the depolarization ratio of the scattered light. The experimental data in support of our predictions are quoted.

\section{General formulae}

The concept of multiplicity arises in the theory of molecular light scattering whenever the iteration procedure is used to solve the integral equation describing the electromagnetic wave propagation in a statistically inhomogeneous medium. In the case of a fluid whose inhomogeneity is caused by the permittivity fluctuations $\delta \epsilon(\mathbf{r})=\varepsilon(\mathbf{r})-\varepsilon_{0}$ about the equilibrium value $\varepsilon_{0}$, this equation takes the form

$$
\mathbf{E}(\mathbf{R})=\mathbf{E}_{0}(\mathbf{R})-k_{0}^{2} \int_{V} \mathrm{~d} \mathbf{r} \widehat{T}(\mathbf{R}-\mathbf{r}) \delta \epsilon(\mathbf{r}) \mathbf{E}(\mathbf{r}),
$$

where $\mathbf{E}(\mathbf{r})$ is the electric field at point $\mathbf{r}$ of the fluid, $\mathbf{E}_{0}(\mathbf{r})=\mathbf{e}_{0} E_{0} \exp (\mathrm{ik} \cdot \mathbf{r})$ is the electric field of the incident wave, $\mathbf{e}_{0}$ is the unit polarization vector, $k_{0}=2 \pi / \lambda_{0}$ is the wave number in vacuum, $k=k_{0} \sqrt{\varepsilon_{0}}$ is the wave number in the fluid, and

$$
T_{\alpha \beta}(r)=-\left(k^{2} \delta_{\alpha \beta}+\nabla_{\alpha} \nabla_{\beta}\right) \frac{\mathrm{e}^{\mathrm{i} k r}}{4 \pi k^{2} r}
$$

stands for the electromagnetic field propagator; $\delta_{\alpha \beta}$ is the Kronecker delta. The time dependence in the above formulae is omitted, for we restrict ourselves to the static picture of scattering.

The application of the iteration procedure to equation (1) leads to the series

$$
\mathbf{E}^{\prime}(\mathbf{R})=\mathbf{E}^{(1)}(\mathbf{R})+\mathbf{E}^{(2)}(\mathbf{R})+\mathbf{E}^{(3)}(\mathbf{R})+\cdots
$$

for the scattered field $\mathbf{E}^{\prime}(\mathbf{R})$, where the first three members are

$$
\begin{aligned}
\mathbf{E}^{(1)}(\mathbf{R})= & -k_{0}^{2} \int_{V} \mathrm{~d} \mathbf{r}_{1} \widehat{T}\left(\mathbf{R}-\mathbf{r}_{1}\right) \delta \epsilon\left(\mathbf{r}_{1}\right) \mathbf{E}_{0}\left(\mathbf{r}_{1}\right), \\
\mathbf{E}^{(2)}(\mathbf{R})= & k_{0}^{4} \int_{V} \mathrm{~d} \mathbf{r}_{2} \int_{V} \mathrm{~d} \mathbf{r}_{1} \widehat{T}\left(\mathbf{R}-\mathbf{r}_{2}\right) \widehat{T}\left(\mathbf{r}_{2}-\mathbf{r}_{1}\right) \delta \epsilon\left(\mathbf{r}_{2}\right) \delta \epsilon\left(\mathbf{r}_{1}\right) \mathbf{E}_{0}\left(\mathbf{r}_{1}\right), \\
\mathbf{E}^{(3)}(\mathbf{R})= & -k_{0}^{6} \int_{V} \mathrm{~d} \mathbf{r}_{3} \int_{V} \mathrm{~d} \mathbf{r}_{2} \int_{V} \mathrm{~d} \mathbf{r}_{1} \widehat{T}\left(\mathbf{R}-\mathbf{r}_{3}\right) \widehat{T}\left(\mathbf{r}_{3}-\mathbf{r}_{2}\right) \widehat{T}\left(\mathbf{r}_{2}-\mathbf{r}_{1}\right) \\
& \times \delta \epsilon\left(\mathbf{r}_{3}\right) \delta \epsilon\left(\mathbf{r}_{2}\right) \delta \epsilon\left(\mathbf{r}_{1}\right) \mathbf{E}_{0}\left(\mathbf{r}_{1}\right) .
\end{aligned}
$$

The meaning of the individual member $\mathbf{E}^{(n)}$ is easy to grasp if we represent (3) graphically, as in figure 1. 


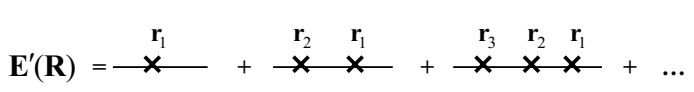

Figure 1. Graphic representation of the scattered field. The rightmost line corresponds to the incident field $\mathbf{E}_{0}\left(\mathbf{r}_{1}\right)$ at point $\mathbf{r}_{1}$ where the first scattering event occurs. The leftmost line represents the propagator $\widehat{T}\left(\mathbf{R}-\mathbf{r}_{n}\right)$ describing the wave propagation from point $\mathbf{r}_{n}$, at which the (last) $n$th scattering event occurs, to the observation point $\mathbf{R}$. The segments between neighboring vertexes $\mathbf{r}_{i+1}, \mathbf{r}_{i}$ stand for the internal propagators $\widehat{T}\left(\mathbf{r}_{i+1}-\mathbf{r}_{i}\right)$ that describe the wave propagation from point $\mathbf{r}_{i}$ to point $\mathbf{r}_{i+1}$ within the system. The vertex $\mathbf{r}_{i}$ is assigned the multiplier $-k_{0}^{2} \delta \epsilon\left(\mathbf{r}_{i}\right)$, and the integrals are taken over all $\mathbf{r}_{i}$.
It is seen that $\mathbf{E}^{(1)}$ is the contribution to the overall scattered field due to a single scattering event, $\mathbf{E}^{(2)}$ describes the contribution from two successive scattering events, etc.

In view of the representation (3), the study of the scattering intensity reduces to that of the tensor

$$
I_{\alpha \beta}=\sum_{n, m \geqslant 1} I_{\alpha \beta}^{n m}
$$

where the correlators

$$
I_{\alpha \beta}^{n m} \propto\left\langle E_{\alpha}^{(n)}(\mathbf{R}) \cdot E_{\beta}^{(m) *}(\mathbf{R})\right\rangle
$$

are defined as the intensities of the scattering effects of multiplicities $(n+m) / 2$, and the subscripts $\alpha$ and $\beta$ determine the polarization states under study. The angle brackets in (8) denote averaging over the statistic of fluctuations $\delta \varepsilon$.

The term $I_{\alpha \beta}^{11}$ is the single scattering intensity, and the terms $I_{\alpha \beta}^{n m}$ with $n \neq m$ describe the socalled interference effects. In particular, the terms $I_{\alpha \beta}^{12}, I_{\alpha \beta}^{21}$ are responsible for the 1.5-multiplicity effects, the main subject of our discussion. In order to estimate them, we should, taking into account the aforesaid, concentrate on the short-range contributions to the intensities $I_{\alpha \beta}^{n m}$. These are caused by: (1) successive re-emission events within a group of closely situated scattering centres, with all the centre-to-centre distances $\left|\mathbf{r}_{i}-\mathbf{r}_{j}\right| \lesssim r_{\mathrm{c}} \ll \lambda$ (such a group is referred to as compact); (2) short-range correlation effects between close pairs of compact groups.

\section{Short-range effects}

\subsection{Short-range re-emission events}

Within a macroscopic approach, a compact group of fluctuations can be treated as a single fluctuation heterogeneity characterized by an effective permittivity $\delta \epsilon_{\alpha \beta}(\mathbf{r})$. Andreev [13] took advantage of this idea to develop the light depolarization mechanism due to the fluctuations of the thermal fluctuation distribution function. The effective permittivity was found in [13] by averaging (direct integrating) the electric field over a macroscopic volume $U$ of linear size $U^{1 / 3} \ll \lambda$, in the same way as the permittivity of a slightly heterogeneous suspension is found [14], and then the Gaussian statistic for the order-parameter fluctuations was employed to calculate the depolarized scattering intensity. It was shown later [3,15-17] in the context of the multiple scattering theory that the Andreev mechanism is basically associated with the scattering by compact pairs of the order-parameter fluctuations. This means that those domains of the integration variables $\mathbf{r}_{i}$ come into play where the arguments of the internal propagators $\widehat{T}\left(\left|\mathbf{r}_{i+1}-\mathbf{r}_{i}\right|\right)$ are small, so that these propagators exhibit singular behaviour.

The latter fact underlies our approach to the analysis of short-range re-emission contributions. The idea is to make use of the methods of the theory of generalized function in order to single out the leading short-range re-emission contributions in the iteration series (3). For this purpose, we prove [11] that for the set of smooth functions $\varphi(\mathbf{r})$ that are constant at zero and are such that $\varphi(\mathbf{r})$, $\partial \varphi(\mathbf{r}) / \partial r$, and $r \partial \varphi(\mathbf{r}) \partial r$ all vanish as $r \rightarrow \infty$, the propagator $T_{\alpha \beta}(r)$ admits the representation

$$
\widetilde{T}_{\alpha \beta}(r)=\frac{1}{3 k^{2}} \delta(\mathbf{r}) \delta_{\alpha \beta} \mathrm{e}^{\mathrm{i} k r}+\frac{1}{4 \pi k^{2}}\left(\frac{1}{r^{3}}-\frac{\mathrm{i} k}{r^{2}}\right)\left(\delta_{\alpha \beta}-3 e_{\alpha} e_{\beta}\right) \mathrm{e}^{\mathrm{i} k r}-\frac{1}{4 \pi r}\left(\delta_{\alpha \beta}-e_{\alpha} e_{\beta}\right) \mathrm{e}^{\mathrm{i} k r}
$$


where $\delta(\mathbf{r})$ is the Dirac delta function, and $e_{\alpha}$ is the $\alpha$ component of the unit vector $\mathbf{e}=\mathbf{r} / r$. This representation should be understood in the sense that the equality

$$
\int_{V} \mathrm{~d} \mathbf{r} T_{\alpha \beta}(r) \varphi(\mathbf{r})=\int_{V} \mathrm{~d} \mathbf{r} \widetilde{T}_{\alpha \beta}(r) \varphi(\mathbf{r})
$$

is valid for any $\varphi(\mathbf{r})$.

The last term in (9) corresponds to distant re-emissions between the scattering centres; it is responsible for the true multiple scattering effects. The other terms describe the short-range behaviour of the propagator. Of these, the second one remains nonzero at $\alpha \neq \beta$ and can therefore result in the depolarization of the scattered light. Our attention, however, is directed to the first term in (9), which is the most singular and affects only polarized scattering.

Replacing the internal propagators with their most singular parts, we find

$$
\mathbf{E}^{(n)}(\mathbf{R})=-k_{0}^{2} \int_{V} \mathrm{~d} \mathbf{r}_{1} \widehat{T}\left(\mathbf{R}-\mathbf{r}_{1}\right)\left(-\frac{1}{3 \varepsilon_{0}}\right)^{n-1}\left(\delta \epsilon\left(\mathbf{r}_{1}\right)\right)^{n} \mathbf{E}_{0}\left(\mathbf{r}_{1}\right) .
$$

It follows immediately that the leading contribution made by short-range re-emissions within an $n$-member compact group to the scalar part of the effective permittivity is

$$
\delta \epsilon_{\alpha \beta}^{(n)}(\mathbf{r})=\left(-\frac{1}{3 \varepsilon_{0}}\right)^{n-1}(\delta \varepsilon(\mathbf{r}))^{n} \delta_{\alpha \beta}
$$

The relevant part of the scattered field is depicted in figure 2 .

It is given by a series similar to (3), in which the term $\mathbf{E}^{(n)}(n \geqslant 2)$ now represents the contribution (11) from short-range re-emissions within an $n$-member compact group. Physically, scattering by such a group is single.

Replacing the propagator $\widehat{T}\left(\mathbf{R}-\mathbf{r}_{n}\right)$ with its wave-zone asymptote, we find in the standard way [14] that only polarized scattering occurs (the polarization subscripts $\alpha$ and $\beta$ are both equal to that in the incident wave). The corresponding scattering intensity takes the form

$$
\begin{aligned}
I & =\sum_{n, m \geqslant 1} I^{n m}, \\
I^{n m} & \propto\left(-\frac{1}{3 \varepsilon_{0}}\right)^{n+m-2} \int_{V} \mathrm{~d} \mathbf{r}\left\langle(\delta \varepsilon(\mathbf{r}))^{n}(\delta \varepsilon(\mathbf{0}))^{m}\right\rangle \mathrm{e}^{-\mathbf{i q} \cdot \mathbf{r}} .
\end{aligned}
$$

Here, $\mathbf{q}$ is the change in the wave-number vector due to the scattering, the subscripts $\alpha, \beta$ are omitted, and the proportionality coefficient is

$$
I_{0} \frac{k_{0}^{4} V}{16 \pi^{2} R^{2}}\left[1-\left(\widehat{\mathbf{R}} \cdot \mathbf{e}_{0}\right)^{2}\right]
$$

where $I_{0}$ is the intensity of the incident light, $V$ is the scattering volume, and $\widehat{\mathbf{R}}=\mathbf{R} / R$ is the unit vector toward the observation point $\mathbf{R}$.

It is not difficult to see that the intensity of scattering by compact groups can be expressed in terms of the spatial Fourier transforms of the irreducible (fluctuation) correlators $\left\langle\left\langle\varepsilon^{n}(\mathbf{r}) \varepsilon^{m}(\mathbf{0})\right\rangle\right\rangle$. In the immediate vicinity of the critical point, where the permittivity fluctuations $\delta \varepsilon(\mathbf{r})$ reduce to the order-parameter fluctuations $\delta \varphi(\mathbf{r})$ by $\delta \varepsilon(\mathbf{r})=(\partial \varepsilon / \partial \varphi) \delta \varphi(\mathbf{r})$, these correlators reduce to the irreducible (fluctuation) correlators of quantities $\varphi^{n}(\mathbf{r})$. The fact of the existence of such a set of fluctuating quantities in the problem of molecular light scattering in fluids near the critical point is in complete agreement with the local-algebra hypothesis [12]. But it is the region of short-range correlations between the compact groups to which we turn our attention. 


\subsection{Short-range correlation contributions}

At a certain distance from the critical point, where non-local correlation between fluctuations is relatively weak, the intensities $I^{n m}$ are determined by the $(n+m)$-order moments $\left\langle(\Delta \varepsilon)^{n+m}\right\rangle$ of thermodynamic permittivity fluctuations [11]:

$$
I^{n m} \propto\left(-\frac{1}{3 \varepsilon_{0}}\right)^{n+m-2} \tilde{V}\left\langle(\Delta \varepsilon)^{n+m}\right\rangle,
$$

where $\widetilde{V}$ is a macroscopic volume over which the averaging is carried out.

In order to prove this fact, we note that the integrals (14) are actually taken over the regions of linear size $r \lesssim r_{\mathrm{c}} \ll \lambda$, so that the exponential factor can be set to unity. Then we define the thermodynamic contributions to the fluctuations of the quantities $\varepsilon^{n}(\mathbf{r})$ by

$$
\Delta(\varepsilon)^{n}=\varepsilon^{n}-\overline{\varepsilon^{n}}=\frac{1}{\widetilde{V}} \int_{\widetilde{V}} \mathrm{~d} \mathbf{r} \delta\left(\varepsilon^{n}(\mathbf{r})\right)
$$

and rewrite the integrals in (14) as

$$
\frac{1}{\widetilde{V}} \int_{\widetilde{V}} \int_{\widetilde{V}} \mathrm{~d} \mathbf{r}_{1} \mathrm{~d} \mathbf{r}_{2}\left\langle\left(\delta \varepsilon\left(\mathbf{r}_{1}\right)\right)^{n}\left(\delta \varepsilon\left(\mathbf{r}_{2}\right)\right)^{m}\right\rangle,
$$

whence formula (15) follows after some manipulations. We exemplify those by considering the intensity $I^{31}$. The pertinent correlator can be written as

$$
\left.\left\langle\left(\delta \varepsilon\left(\mathbf{r}_{1}\right)\right)^{3} \delta \varepsilon\left(\mathbf{r}_{2}\right)\right\rangle=\left\langle\delta\left(\varepsilon^{3}\left(\mathbf{r}_{1}\right)\right) \delta \varepsilon\left(\mathbf{r}_{2}\right)\right\rangle-3 \bar{\varepsilon}\left\langle\delta\left(\varepsilon^{2}\left(\mathbf{r}_{1}\right)\right) \delta \varepsilon\left(\mathbf{r}_{2}\right)\right\rangle+3 \bar{\varepsilon}^{2}\left\langle\delta \varepsilon\left(\mathbf{r}_{1}\right)\right) \delta \varepsilon\left(\mathbf{r}_{2}\right)\right\rangle .
$$

If we now integrate it over $\mathbf{r}_{1}$ and $\mathbf{r}_{2}$ and then divide it by $\widetilde{V}^{2}$, it transforms, in view of formula (16), to

the above result.

$$
\overline{\varepsilon^{4}}-4 \overline{\varepsilon^{3}} \bar{\varepsilon}+6 \overline{\varepsilon^{2}} \bar{\varepsilon}^{2}-3 \bar{\varepsilon}^{4}=\left\langle(\Delta \varepsilon)^{4}\right\rangle,
$$

Far away from the critical point, the linear size $\widetilde{V}^{1 / 3}$ should considerably exceed the radius of action of intermolecular force and yet it can remain much smaller than $\lambda$ [14]. In our estimates [11], we took $\widetilde{V}$ to be equal to $2.5 \times 10^{-19} \mathrm{~cm}^{3}$ for correlation lengths $r_{\mathrm{c}} \lesssim 4 \times 10^{-7} \mathrm{~cm}$; in the case of xenon, the latter correspond to reduced temperatures $\tau \gtrsim 1 \times 10^{-2}$. In principle, as the further analysis reveals, this value of $\widetilde{V}$ can safely be increased by an order of magnitude, the new value to be used for $r_{\mathrm{c}} \lesssim 8.5 \times 10^{-7} \mathrm{~cm}\left(\tau \gtrsim 3 \times 10^{-3}\right.$ for xenon).

The value of $\widetilde{V}$ becomes more definite as the critical point is approached: with the correlation length becoming much greater than any linear size characterizing the interaction of the particles in the system, we can set $\widetilde{V} \approx 4 \pi r_{\mathrm{c}}^{3} / 3[12]$.

In a single-component fluid, the permittivity fluctuations are mainly due to the thermodynamic fluctuations of density: $\Delta \varepsilon=(\partial \varepsilon / \partial \rho) \Delta \rho$. In this case, the problem reduces to finding the higher-order moments of the thermodynamic density fluctuations. In particular, the 1.5-scattering intensity is determined by the third-order moment $\left\langle(\Delta \rho)^{3}\right\rangle$.

The formal expressions for the third (non-Gaussian) and fourth-order moments of the density fluctuation can be found using the grand canonical ensemble. Then, retaining in series (13) the terms of up to the fourth order in $\Delta \rho$, and taking into account that the non-Gaussian contribution to the latter moment remains negligible at temperatures currently reachable $[2,10]$, we obtain [11]:

$$
\begin{aligned}
I_{1} & \equiv I_{11} \propto\left(\rho \frac{\partial \varepsilon}{\partial \rho}\right)^{2} k_{\mathrm{B}} T \beta \\
I_{1.5} & \equiv I_{12}+I_{21} \propto-\frac{2}{3 \varepsilon_{0}}\left(\rho \frac{\partial \varepsilon}{\partial \rho}\right)^{3} \frac{k_{\mathrm{B}}^{2} T^{2}}{\widetilde{V}}\left[2 \beta^{2}+\left(\frac{\partial \beta}{\partial P}\right)_{T, V}\right] \\
I_{2} & \equiv I_{13}+I_{22}+I_{31} \propto \frac{1}{\varepsilon_{0}^{2}}\left(\rho \frac{\partial \varepsilon}{\partial \rho}\right)^{4} \frac{k_{\mathrm{B}}^{2} T^{2}}{\widetilde{V}} \beta^{2}
\end{aligned}
$$


where $k_{\mathrm{B}}$ is the Boltzmann constant, $P$ is the pressure, and $\beta$ is the isothermal compressibility of the fluid.

In the above formulae, $I_{1}$ is the intensity of single scattering, $I_{1.5}$ gives the intensity of 1.5 multiplicity scattering, and $I_{2}$ represents the contribution to the double scattering from four closely situated scattering centres. Far enough from the critical point, where $\widetilde{V}$ can be taken to be constant, $I_{2}$ increases as $\beta^{2} \propto r_{\mathrm{c}}^{4}$, that is, by the same law as the intensities of the true-double polarized $\left(I_{d}\right)$ and depolarized $\left(I_{d d}\right)$ scatterings do $[6,15]$ :

$$
I_{d} \propto \frac{1}{15 \pi}\left(\rho \frac{\partial \varepsilon}{\partial \rho}\right)^{4} L k_{0}^{4} k_{\mathrm{B}}^{2} T^{2} \beta^{2}, \quad I_{d d} \sim \frac{1}{8} I_{d}
$$

where $L$ is the linear size of the system in cm. With $\widetilde{V}$ increasing as $r_{\text {c }}^{3}$, we obtain the result of the Gaussian model for order-parament fluctuations [3,16]: $I_{2} \propto r_{\mathrm{c}}$.

Further analysis requires the knowledge of the explicit form for the derivative of $\beta$ with respect to pressure, which is not available at present. Nonetheless, we can infer a number of useful functional relationships and quantitative estimates by restricting ourselves to consideration of its functional structure in the gas phase. For this purpose, taking into account the temperature interval involved, we can try to employ the well-known van der Waals equation of state.

\section{Estimates with the Van der Waals model}

Within the framework of the van der Waals model, we find

$$
\left(\frac{\partial \beta}{\partial P}\right)_{T, V}=-2 \beta^{2}+\frac{3 \omega}{3 \omega+2}\left[\beta^{2}+\frac{6 P_{\mathrm{c}}}{(1+\omega)^{2}} \beta^{3}\right]
$$

where $\omega \equiv \rho_{\mathrm{c}} / \rho-1$ is the deviation of the density from the critical value $\rho_{\mathrm{c}}$, and $P_{\mathrm{c}}$ is the critical pressure. Correspondingly, for small deviations $\omega \ll 1$, taking into account that $\beta \geqslant\left(6 P_{\mathrm{c}} \tau\right)^{-1}$, we have the estimate

$$
I_{1.5} \propto-\frac{1}{\varepsilon_{0}}\left(\rho \frac{\partial \varepsilon}{\partial \rho}\right)^{3} \frac{k_{\mathrm{B}}^{2} T^{2}}{\widetilde{V}} \cdot 6 P_{\mathrm{c}} \omega \beta^{3} .
$$

On the critical isochore $I_{1.5}=0$, which is in accord with the conformal invariance condition [19]. The 1.5-scattering comes into play under the condition of deviating from the critical isochore $(\omega \neq 0)$. If so, then far away from the critical point, where we expect $\widetilde{V}$ to be slightly dependent on the temperature, the 1.5-scattering intensity increases in absolute value as $\beta^{3}$ as the critical point is approached. When $\tau$ becomes less than $3 \times 10^{-3}$ and, according to our assumption, $\widetilde{V} \propto r_{\mathrm{c}}^{3} \propto \beta^{3 / 2}$, the temperature dependence of $I_{1.5}$ is given by

$$
I_{1.5} \propto-\omega \beta^{3 / 2}
$$

Then, in view of formulae (17), (19), (20), and (22), the relative magnitude of $I_{1.5}$, as compared to those of $I_{1}, I_{2}$, and $I_{d}$, changes with temperature as

$$
\frac{\left|I_{1.5}\right|}{I_{1}} \propto|\omega| \beta^{1 / 2}, \quad \frac{\left|I_{1.5}\right|}{I_{2}} \propto|\omega| \beta, \quad \frac{\left|I_{1.5}\right|}{I_{d}} \propto|\omega| \beta^{-1 / 2} .
$$

To estimate the numerical values of the proportionality coefficients, we take advantage of the data [18] for xenon: $\varepsilon_{0} \approx 1.3, \rho \partial \varepsilon / \partial \rho \approx 0.33, P_{\mathrm{c}}=5.84 \times 10^{7} \mathrm{dyn} \cdot \mathrm{cm}^{-2}, \rho_{\mathrm{c}}=188.2$ Amagat, $T_{\mathrm{c}}=289.765 \mathrm{~K}, k_{\mathrm{B}} T \approx 4 \times 10^{-14} \mathrm{erg}$, the correlation length amplitude $r_{0} \approx 2.2 \times 10^{-8} \mathrm{~cm}$. Using now the value 0.5 for the critical exponent $\nu$ in the scaling law $r_{\mathrm{c}}=r_{0} \tau^{-\nu}$, for $\lambda \approx 4.28 \times 10^{-5} \mathrm{~cm}$ and $L=0.547 \mathrm{~cm}$ we obtain:

$$
\frac{\left|I_{1.5}\right|}{I_{1}} \approx 0.65|\omega| \tau^{-1 / 2}, \quad \frac{\left|I_{1.5}\right|}{I_{2}} \approx 3.9|\omega| \tau^{-1}, \quad \frac{\left|I_{1.5}\right|}{I_{d}} \approx 1.6 \times 10^{4}|\omega| \tau^{1 / 2} .
$$


The most significant result of these rather rough estimates is as follows: near the critical isochore, there may exist temperature and density ranges where the 1.5-scattering exceeds the true double scattering and is therefore measurable. In particular, along the isochore $\rho=186.93$ Amagat $\left(\omega=6.8 \times 10^{-3}\right)$, experimental data for which are available in [18], the ratio $\left|I_{1.5}\right| / I_{1}$ increases from 0.14 to 0.44 , the ratio $\left|I_{1.5}\right| / I_{d}$ decreases from 3.4 to 1.1 , and the term $I_{2}$ is negligible as compared to $\left|I_{1.5}\right|$ as $\tau$ decreases from $1 \times 10^{-3}$ to $1 \times 10^{-4}$; the true double scattering starts dominating over the 1.5 -scattering at $\tau \approx 8.4 \times 10^{-5}$. The relative magnitude of the 1.5 -scattering can be increased by increasing $\omega$ and reducing $L$. The fact that $I_{1.5}<0$ for $\omega>0$ (as we shift from the critical isochore to lower densities) becomes of crucial importance for its separation from the total scattering.

\section{Experimental data analysis}

To our knowledge, no specially designed experiment has ever been done on the 1.5-scattering. Nonetheless, our predictions can be tested to a certain extent if we process the data available on the depolarization ratio $\Delta$ of the scattered light. We refer to the experiment by Trappeniers et al. [18] who studied in detail the dependence of $\Delta$ upon the parameter $D=k_{0}^{-4}\left[\left(\varepsilon_{0}-1\right)\left(\varepsilon_{0}+2\right) / 12 \pi\right]^{-2}$ $\times\left(k_{\mathrm{B}} T \beta\right)^{-1}$ for xenon.

In accordance with the preceding consideration, we can write

$$
\Delta=\frac{I_{a}+I_{d d}}{I_{1}+I_{1.5}+I_{d}}
$$

where $I_{a}$ denotes the intensity of scattering due to the anisotropy fluctuations $\left(I_{a}\right.$ is relatively small in simple fluids and is virtually insensitive to the critical point); the contribution $I_{22}$ is omitted. From (26), we can pass to the relation

$$
\frac{I_{d d}}{I_{1} \Delta}=\frac{1+I_{1.5} I_{1}^{-1}+I_{d} I_{1}^{-1}}{1+I_{a} I_{d d}^{-1}}
$$

In view of formulas $(17),(19),(20),(22)$, and the positivity of the contributions $I_{1}, I_{d}, I_{d d}$, and $I_{a}$, its structure is

$$
\frac{\left(k_{\mathrm{B}} T \beta\right)}{\Delta} \propto \frac{1-a \omega \widetilde{V}^{-1}\left(k_{\mathrm{B}} T \beta^{2}\right)+b\left(k_{\mathrm{B}} T \beta\right)}{1+c\left(k_{\mathrm{B}} T \beta\right)^{-2}},
$$

where $a, b$, and $c$ are positive constants.

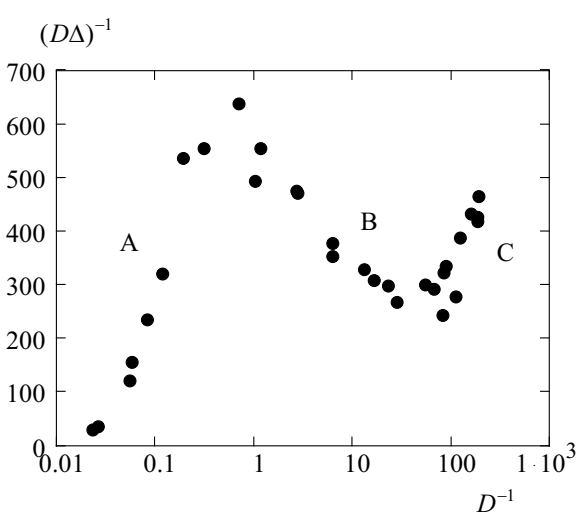

Figure 3. $(D \Delta)^{-1}$ versus $D^{-1}$ along the $\omega=$ $6.8 \times 10^{-3}$ isochore for xenon as $\tau$ decreases from $7.8 \times 10^{-2}$ to $3.5 \times 10^{-5}$, according to data [18]. The maximum and minimum occur at $\tau=4.1 \times$ $10^{-3}$ and $1.8 \times 10^{-4}$ respectively. Three segments $\mathrm{A}, \mathrm{B}$, and $\mathrm{C}$ are clearly distinguished.
In the absence of the 1.5-scattering $(a=0)$, the left side $\left(k_{\mathrm{B}} T \beta\right) / \Delta \propto(D \Delta)^{-1}$ is a monotonically increasing function of $\left(k_{\mathrm{B}} T \beta\right) \propto D^{-1}$ that rapidly becomes linear as the denominator decreases. In the close vicinity of the critical point, where non-local correlation between fluctuations becomes of importance and the growth of the single and the true double scatterings slow down, this function levels off. The presence of the 1.5-scattering $(a \neq 0)$ must result in this monotonic behaviour being violated for $\omega>0$ : after the initial increase, the $(D \Delta)^{-1}$ versus $D^{-1}$ dependence is expected to pass through a maximum, then decrease, reach a minimum, then increase again, and eventually level off.

As figure 3 reveals, the qualitative behaviour of the experimentally-based plot and parameters of its characteristic points are in good agreement with our predictions. 
Segments A, B, and C in figure 3 can further be scrutinized as follows.

As long as the terms $\left|I_{1.5}\right| / I_{1}$ and $I_{d} / I_{1}$ are negligible in comparison with unity, and the sharp growth in $\left(k_{\mathrm{B}} T \beta\right) / \Delta$ is indeed caused by the denominator, formula $(28)$ can be rewritten as $\Delta /\left(k_{\mathrm{B}} T \beta\right) \propto$ const $+\left(k_{\mathrm{B}} T \beta\right)^{-2}$. So, a linear dependence of $(D \Delta)$ on $D^{2}$ is expected for segment A (see figure 4).

If we ignore $I_{a} / I_{d d}$ and $I_{d} / I_{1}$ for temperatures $6.5 \times 10^{-4} \lesssim \tau \lesssim 1.8 \times 10^{-4}$, where the decrease in $\left(k_{\mathrm{B}} T \beta\right) / \Delta$ is possibly due to the 1.5-scattering and $\widetilde{V} \propto r_{\mathrm{c}}^{3} \propto \beta^{3 / 2}$, then formula (28) yields $\beta / \Delta \propto$ const $-\beta^{1 / 2}$. A linear dependence of $(D \Delta)^{-1}$ on $-D^{-1 / 2}$ is expected for this part of segment B (see figure 5).

Finally, with the true double scattering prevailing over the 1.5 scattering, (28) changes to $\beta / \Delta \propto$ const $+\beta$. A linear dependence of $(D \Delta)^{-1}$ upon $D^{-1}$ is expected for segment $\mathrm{C}$ (see figure 6).

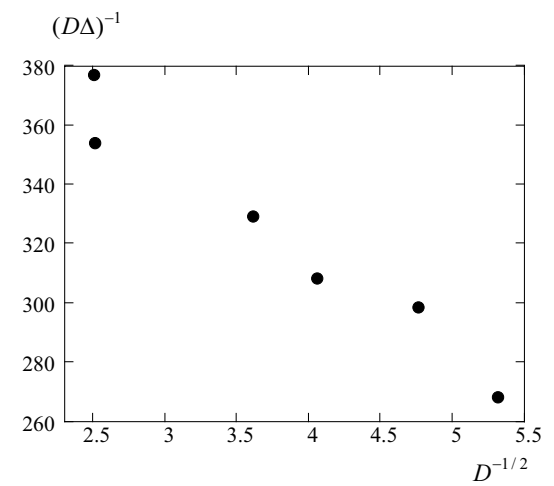

Figure 5. $(D \Delta)^{-1}$ versus $D^{-1 / 2}$ for the portion of segment $\mathrm{B}$ where $\tau$ decreases rightwards from $6.5 \times 10^{-4}$ to $1.8 \times 10^{-4}$. Take note of the negative slope.

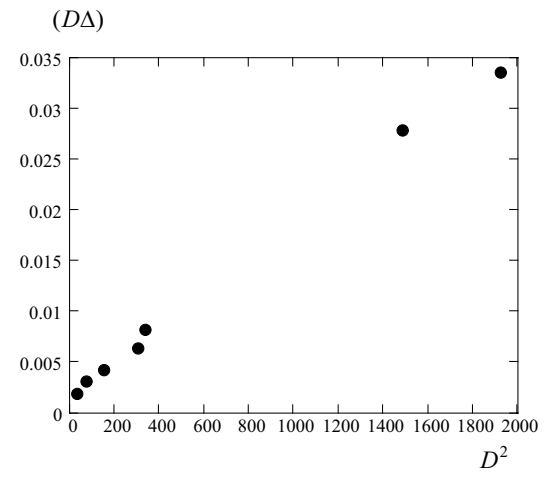

Figure 4. $(D \Delta)$ versus $D^{2}$ for segment A. From right to left, $\tau$ decreases from $7.8 \times 10^{-2}$ to $4.1 \times$ $10^{-3}$.

As figures 4, 5, and 6 reveal, all the three linear relationships seem to occur.

\section{Conclusion}

The presence of the 1.5-scattering should significantly enrich and complicate the overall scattering pattern in fluids near the critical point. Several other effects can be shown to become possible in addition to those mentioned above: (1) far enough from the critical point, the 1.5-scattering alone causes the depolarization ratio to increase with temperature by the law $\Delta \propto \beta$, typical of the true double scattering mechanism; (2) the joint action of the 1.5-scattering and the true double scattering mechanisms causes the divergence of $\Delta$ with $\beta$ to become stronger in the temperature range $\tau \lesssim 10^{-3}$ (such an increase was observed in [7], but was attributed to triple light scattering); (3) subsequently, the increase of $\Delta$ may change to a decrease at $\tau \lesssim 10^{-4}$ (such a decrease was indeed observed in [20], but was subjected to question). The details of the pertinent analysis will be published elsewhere. 
We hope that the theoretical findings and experimental facts presented in this report are strong enough to lend impetus to specially planned experiments on the 1.5-scattering effects. The confirmation of our conclusions would signify that the method of molecular spectroscopy offers a certain opportunity of measuring the third-order fluctuation moments and related parameters of the fluid.

\title{
References
}

1. Oxtoby D.W., Gelbart W.M., J. Chem. Phys., 1974, 60, 3359.

2. Lakoza E.L., Chalyi A.V., Zh. Éksp. Teor. Fiz., 1974, 67, 1050 (in Russian); Sov. Phys. JETP, 1975, 40, 521.

3. Adzhemyan L.V, Adzhemyan L.Ts., Zubkov L.A., Romanov V.P., Pis'ma Zh. Éksp. Teor. Fiz., 1975, 22, 11 (in Russian); JETP Lett., 1975, 22, 5.

4. Kuzmin V.L, Optika i Spektr., 1975, 38, 745 (in Russian).

5. Boots H.M.J., Bedeaux D., Mazur P., Physica A, 1975, 79, 397.

6. Lakoza E.L., Chalyi A.V., Usp. Fiz. Nauk, 1983, 140, 393 (in Russian); Sov. Phys. Usp., 1983, 26, 573.

7. Trappeniers N.J., Huijser R.H., Michels A.C., Chem. Phys. Lett., 1977, 48, 31.

8. Lebowitz J.L., Percus J.K., Phys. Rev., 1961, 122, 1675.

9. Kuni F.M., Dokl. AN SSSR, 1968, 179, 129 (in Russian).

10. Sushko M.Ya., Ukr. Fiz. Zh., 2004, 49, 712 (in Ukrainian); Ukr. J. Phys., 2004, 49, 710.

11. Sushko M.Ya., Zh. Éksp. Teor. Fiz., 2004, 126, 1355 (in Russian); JETP, 2004, 99, 1183.

12. Patashinskiı A.Z., Pokrovskï V.L. Fluctuation Theory of Phase Transitions, 2nd ed. Nauka, Moscow, 1982 (in Russian); Pergamon Press, Oxford, 1979.

13. Andreev A.F, Pis'ma Zh. Éksp. Teor. Fiz., 1974, 19, 713 (in Russian); JETP Lett., 1974, 19, 368.

14. Landau L.D., Lifshitz E.M., Course of Theoretical Physics, Vol. 8: Electrodynamics of Continuous Media, 2nd ed. Nauka, Moscow, 1982 (in Russian); Pergamon Press, Oxford, 1984.

15. Malomuzh N.P, Sushko M.Ya., Ukr. Fiz. Zh., 1985, 30, 363 (in Russian).

16. Malomuzh N.P, Sushko M.Ya., Zh. Éksp. Teor. Fiz., 1985, 89, 435 (in Russian); Sov. Phys. JETP, 1985, 62, 246.

17. Sushko M.Ya., Ukr. Fiz. Zh., 1986, 31, 679 (in Russian).

18. Trappeniers N.J., Michels A.C., Boots H.M.J., Huijser R.H., Physica A (Amsterdam), 1980, $101,431$.

19. Polyakov A.M., Pis'ma Zh. Éksp. Teor. Fiz., 1970, 12, 538 (in Russian); JETP Lett., 1970, 12, 381.

20. Trappeniers N.J., Michels A.C., Huijser R.H., Chem. Phys. Lett., 1975, 34, 192.

\section{Молекулярне розсіяння світла кратності 1.5 у рідинах}

\author{
М.Я.Сушко \\ Одеський національний університет ім. І.І.Мечникова, \\ вул. Дворянська, 2, Одеса 65026, Україна
}

Отримано 25 червня 2005 р.

Показано, що третій момент термодинамічних флуктуацій густини вносить помітний внесок у молекулярне розсіяння світла в однокомнонентній рідині. Пов'язане з ним розсіяння кратності 1.5 повинно впливати на значення коефіцієнта деполяризації розсіяного світла, якщо розсіяння вивчається поблизу критичної ізохори та в тому температурному інтервалі, де нелокальні кореляції між флуктуаціями є відносно малими. Наводяться експериментальні дані на користь цього висновку.

Ключові слова: флуктуації густини, третій момент, розсіяння світла

PACS: $05.40,05.70 . F h, 64.70 . F x, 78.35 .+C$ 
